\title{
Nitric oxide in occurrence, progress and therapy of lung Cancer: a systemic review and meta-analysis
}

Hongbin Zhou ${ }^{1+}$, Jiuke $\mathrm{Li}^{2+}$, Zhewen Chen ${ }^{3}$, Ying Chen ${ }^{3}$ and Sa Ye ${ }^{1,3^{*}}$

\begin{abstract}
Background: Nitric oxide (NO) plays an important role in lung cancer. However, the results of previous studies about NO in the occurrence, progress and therapy were not consistent. Therefore, we conducted a meta-analysis to evaluate the relationship between $\mathrm{NO}$ and lung cancer.

Method: We carried out comprehensive search in the databases, and collected related studies. The data of fraction of exhaled nitric oxide (FeNO) or blood $\mathrm{NO}$ in different populations (lung cancer patients and control subjects) and different time points (before therapy and after therapy) were extracted by two investigators. A random effect model was applied to analyze the differences of FeNO and blood NO in different populations and different time points. To further compare NO level of each subgroup with different pathological types and different stages, a network metaanalysis (NMA) was performed.
\end{abstract}

Results: Fifty studies including 2551 cases and 1691 controls were adopted in this meta-analysis. The FeNO (SMD $3.01,95 \% \mathrm{Cl} 1.89-4.13, p<0.00001)$ and blood NO (SMD 1.34, 95\% Cl 0.84-1.85, $p<0.00001$ ) level in lung cancer patients was much higher than that in control subjects. NMA model indicated blood NO level in each cancer type except SCLC was higher than that in control patients. There was no significant difference of blood NO level among four kinds of lung cancer patients. Blood NO level in LCC patients (SUCRA $=83.5 \%)$ was the highest. Blood NO level in advanced stage but not early stage was higher than that in control subjects. Patients in advanced stage (SUCR $\mathrm{A}=95.5 \%$ ) had the highest blood NO level. No significant difference of FeNO (SMD $-0.04,95 \% \mathrm{Cl}-0.46-0.38, p>$ $0.05)$ and blood NO level (SMD $-0.36,95 \% \mathrm{Cl}-1.08-0.36, p>0.05)$ was observed between pretreatment and posttreatment in all patients. However, FeNO level elevated (SMD 0.28, 95\% Cl 0.04-0.51, $p=0.02$ ) and blood NO level decreased in NSCLC patients (SMD -0.95, 95\% Cl -1.89-0.00, $p=0.05$ ) after therapy.

Conclusion: FeNO and blood NO level would contribute to diagnosis of lung cancer and evaluation of therapy effect, especially for NSCLC patients.

Keywords: Nitric oxide (NO), Fraction of exhaled nitric oxide (FeNO), Lung cancer

\footnotetext{
* Correspondence: yesa@hmc.edu.cn

${ }^{+}$Hongbin Zhou and Jiuke Li contributed equally to this work.

'Department of Respiratory Medicine, Department of Nutrition, Zhejiang

Provincial People's Hospital, Affiliated People's Hospital, Hangzhou Medical

College, 158 Shangtang Road, Hangzhou 310014, China

${ }^{3}$ Department of Nutrition, Zhejiang Provincial People's Hospital, Affiliated

People's Hospital, Hangzhou Medical College, Zhejiang, Hangzhou, China

Full list of author information is available at the end of the article
}

\section{$\triangle B M C$}

(c) The Author(s). 2021 Open Access This article is licensed under a Creative Commons Attribution 4.0 International License, which permits use, sharing, adaptation, distribution and reproduction in any medium or format, as long as you give appropriate credit to the original author(s) and the source, provide a link to the Creative Commons licence, and indicate if changes were made. The images or other third party material in this article are included in the article's Creative Commons licence, unless indicated otherwise in a credit line to the material. If material is not included in the article's Creative Commons licence and your intended use is not permitted by statutory regulation or exceeds the permitted use, you will need to obtain permission directly from the copyright holder. To view a copy of this licence, visit http://creativecommons.org/licenses/by/4.0/ The Creative Commons Public Domain Dedication waiver (http://creativecommons.org/publicdomain/zero/1.0/) applies to the data made available in this article, unless otherwise stated in a credit line to the data. 


\section{Introduction}

Lung cancer, a kind of tumor with the highest incidence, is also the leading cause of cancer death in the world [1]. It was estimated that about 1.76 million patients died from lung cancer in 2018 globally [2]. The pathogenesis of lung cancer is considered as a multi-stage process, which is affected by genetic background and environmental factors [3]. So far, the underlying mechanisms have not been fully understood. However, it has been widely accepted that oxidative/nitrative damage is the crucial component element leading to carcinogenesis.

Nitric oxide (NO), a small molecule derived from Larginine, has been demonstrated to participate in inflammation, tumor immunity and tumor apoptosis, as well as other pathophysiological process, which are associated with the pathogenesis, progression of lung cancer [4]. NO is produced by airway epithelial cells, vascular endothelial cells, resident macrophages and recruiting inflammatory cells in respiratory system. A part of $\mathrm{NO}$ in the airway will be expired through breath movement, and the fraction of exhaled nitric oxide (FeNO) can be measured by a specific equipment. The value of FeNO is considered as an indicator of airway inflammation level and applied in other chronic respiratory diseases, such as asthma and COPD [5]. In spite of gaseous form, NO also exists in the forms of nitrate or nitrite in circulatory system. The concentration of nitrate or nitrite in peripheral blood can be detected to evaluate systemic NO level.

During the past several decades, many researchers carried out a series of studies try to clarify the relationship between NO and lung cancer. These studies were mainly about comparisons of the levels of FeNO and blood NO between lung cancer patients and healthy controls [645], among lung cancer patients with different stages [7-9, 14, 17-21, 23, 27, 29, 31, 34, 36-39, 42] or types $[6,8,9,18-21,24,26,27,29,31,37-39,42]$, and variations of these indexes from pretreatment to posttreatment [7, 10, 14, 19, 21, 29, 32, 34, 36, 38, 39, 43, 46-55]. However, the results were not consistent among different studies, which might be due to ethnicity, age, pathological type, cancer stage, treatment regimen and follow up period. So, we conducted this meta-analysis to investigate the role of $\mathrm{NO}$ in occurrence, progress and therapy of lung cancer.

\section{Methods}

\section{Search strategy}

We adopted a comprehensive search strategy in databases, including PubMed, Embase and CNKI, to identify the studies about NO and lung cancer. The terms we used were as follows:" lung cancer"," nitric oxide", "NO", "fraction of exhaled nitric oxide", "FeNO". Additional studies were identified by a manual search from references of original studies or review articles on this topic.

\section{Study selection}

The criteria for the selection of studies were as follows:

(1) Case-control studies with control group and at least one case group consisting of lung cancer patients; (2) Other observational studies about comparison between pretreatment and posttreatment, regardless of concrete therapeutic regimen; (3) Case-control studies should provide $\mathrm{FeNO}$ or blood NO data from both control group and case group; (4) Other observational studies should provide FeNO or blood NO data collected before and after treatment. The studies including other kinds of cancers, in which the data of lung cancer could not be obtained alone, were excluded from our analysis.

\section{Data extraction}

The data were independently extracted from all eligible publications by two authors according to the inclusion criteria listed above. Any disagreements were resolved by discussions with a third person. The information extracted from all the studies included author, publication year and observed indexes (FeNO, blood NO). For casecontrol studies, the traits of enrolled patients and control subjects (quantity, nationality, sex, age, smoking status), and cancer characteristics (histological type and stage) were also involved. For other observational studies, the basic and therapy information (quantity, nationality, sex, age, histological type, stage, regimen and follow up period) of enrolled patients were recorded.

\section{Quality assessment}

All the included studies were assessed with the help of Newcastle-Ottawa Scale (NOS), which was mainly about three aspects composed of selection, comparability and exposure [56]. Each study was assigned a score from 0 to 9 points, and higher points indicated higher quality.

\section{Statistical analysis}

Standard mean difference (SMD) and 95\% confidence intervals (CIs) were used to assess the differences of FeNO and blood NO concentrations between control subjects and lung cancer patients, or between pretreatment and posttreatment.

$\mathrm{I}^{2}$ statistic was used to quantify the degree of heterogeneity, with $\mathrm{I}^{2}<25,25-75 \%$ and $>75 \%$ representing low, moderate and high degrees of inconsistency, respectively $[57,58]$. In the analysis of pooled data, we used two different models according to the traits of the included studies: If no heterogeneity was found, a fixed effect model was adopted to determine the effects or a random effect model was applied. If heterogeneity existed across studies, a subgroup analysis was 
performed to seek out the source of heterogeneity. The studies were subdivided by nationality (eastern countries vs. western countries or China vs. Non-China), Age (matched vs. unmatched vs. unknown), sex proportion (matched vs. unmatched vs. unknown), histological types (NSCLC vs. NSCLC + SCLC vs. unknown), stages (early + advanced vs. early vs. advanced vs. unknown), treatment (surgery vs. chemotherapy vs. radiotherapy vs. other therapy), and follow up period (long term vs. short term vs. unknown).

To further compare NO level of each subgroup with different pathological types and different stages, a network meta-analysis (NMA) was conducted. NMA was performed through a Bayesian random-effect model with Markov Chain Monte Carlo simulations which was executed by the 'gemtc' package in R. Using four parallel chains, 50,000 samples after 20,000-sample burn-in were obtained in each chain. Convergence of the model was evaluated with the use of Brooks-Gelman-Rubin diagnostic method in the 'coda' package in R. Consistency of the NMA, defined as a statistical discrepancy between direct and indirect comparison results, was assessed with the help of a node-splitting approach in the 'gemtc' package in $\mathrm{R}$.

The model ranked each pathological type or stage by their relative effect (probabilities of being higher). $\mathrm{Cu}$ mulative probability of being the pathological type or stage with the highest NO level was calculated. With that, the surface under the cumulative ranking curve (SUCRA) of each pathological type or stage is obtained [59]. Specifically, SUCRA is a numeric presentation of the overall ranking and presents a single number, ranging from 0 to $100 \%$, associated with each subgroup, where $0 \%$ represents the lowest NO level and $100 \%$ represents the highest NO level.

We made use of a Begg's funnel plot to examine the underlying publication bias and Egger's weighted regression method to calculate a $P$ value for bias $[60,61]$. If no publication bias exists, the funnel plot appears symmetrical.

All analyses were conducted with the use of Review Manager, V.5.2 (Revman, The Cochrane Collaboration, London, UK), STATA software, V.14.0 (StataCorp LP, College Station, TX, USA) and R software, V.4.0.3.

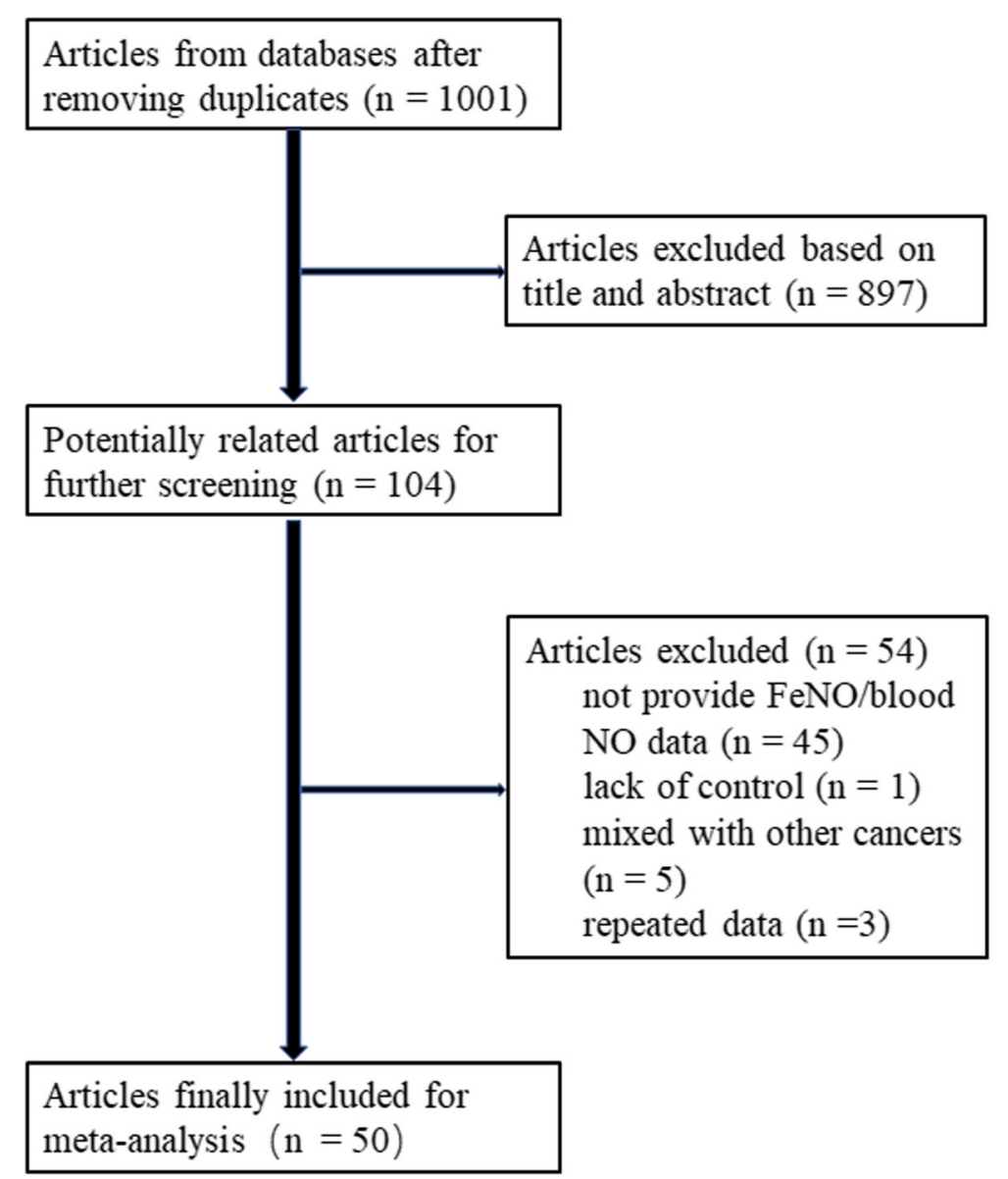

Fig. 1 The flowchart of the study selection process for the meta-analysis 


\section{Results}

\section{Characteristics of the included studies}

We identified 1001 related articles, of which 104 studies were potentially suitable. Forty-five studies were eliminated because they provided neither FeNO nor blood NO data. One study was excluded due to lack of control subjects. Five studies were ruled out because these studies contained different kinds of cancer patients, and the data of lung cancer could not be obtained. In addition, 3 repeated studies were also discarded. Finally, 50 studies including 2551 cases and 1691 controls met the including criteria (Fig. 1). There were 40 case-control studies, in which 6 studies were about FeNO, while 34 studies showed blood NO data. Seven studies provided FeNO data, while 15 studies provided blood NO data collected before and after treatment, respectively. The study characteristics were listed in Table 1, 2, 3 and 4. Lung cancer patients were diagnosed by operation or biopsy. Frequency-matched controls to the cases by sex, age and smoking status were applied in some studies. The treatment regimen mentioned in these studies included operation, chemotherapy, radiotherapy, immunotherapy and traditional Chinese medicine. The scores of included studies ranged from 5 to 9 by NOS.

\section{FeNO level between lung cancer patients and control subjects}

There were 6 case-control studies about FeNO level. The FeNO level in lung cancer patients was much higher than that in control subjects (SMD 3.01, 95\% CI 1.89-4.13, $p<0.00001$ ) (Fig. 2a).

\section{FeNO level between pretreatment and posttreatment}

Seven observational studies reported FeNO level detected before and after therapy. As a result, no significant difference of FeNO level was observed between pretreatment and posttreatment (SMD -0.04, 95\% CI -0.46-0.38, $p>0.05$ ) (Fig. 2b). However, FeNO level elevated in NSCLC subgroup (SMD 0.28, 95\% CI 0.04$0.51, p=0.02$ ) and long-term subgroup (SMD 0.36, 95\% CI $0.11-0.61, p=0.005)$. In other subgroups, there was no evident variance of FeNO level (Table S1).

\section{Blood NO level between lung cancer patients and control subjects}

Thirty-four case-control studies provided data of blood NO level derived from both lung cancer and control groups. Generally, blood NO concentration in lung cancer patients was higher than that in control subjects (SMD 1.34, 95\% CI 0.84-1.85, $p<0.00001$ ) (Fig. 3a). Subgroup analysis indicated this effect existed in almost all subgroups divided by nationality, sex, age and smoking status. However, this effect was only observed in NSCLC subgroup (SMD 2.40, 95\% CI 1.76-3.03, $p<$ 0.00001), while in subgroup containing SCLC patients, there was no difference of blood NO level between lung cancer patients and control subjects (SMD 0.38, 95\% CI -0.25-1.02, $p=0.23)$. The similar phenomenon was also found in subgroups divided by cancer stage. In the subgroup including both early and advanced stage patients, the blood NO level in patients was higher than that in control subjects (SMD 1.60, 95\% CI 0.92-2.27, $p<$ 0.00001 ). For other subgroups (early stage, advanced stage and unknown stage), the difference did not exist (Table S2).

\section{Blood NO level in lung cancer patients with different pathological types and stages}

A total of 16 studies reported the blood NO level of lung cancer patients with different pathological types respectively, including squamous cell carcinoma (SCC), adenocarcinoma (ADC), large cell carcinoma (LCC) and small cell lung cancer (SCLC) (Fig. 4a). To compare the blood NO level in each cancer type, network meta-analysis based on Bayesian random-effect model was applied. As a result, blood NO level in each cancer type except for

Table 1 Characteristics of case-control studies about FeNO

\begin{tabular}{|c|c|c|c|c|c|c|c|c|c|c|c|c|}
\hline \multirow[t]{3}{*}{ Year } & \multirow[t]{3}{*}{ Author } & \multirow[t]{3}{*}{ Country } & \multirow{3}{*}{$\begin{array}{l}\text { Age } \\
\text { matched }\end{array}$} & \multirow{3}{*}{$\begin{array}{l}\text { Sex } \\
\text { matched }\end{array}$} & \multirow{3}{*}{$\begin{array}{l}\text { Smoking } \\
\text { matched }\end{array}$} & \multirow[t]{3}{*}{ Histology } & \multirow[t]{3}{*}{ Stage } & \multicolumn{4}{|l|}{ FeNO (ppb) } & \multirow[t]{3}{*}{ NOS } \\
\hline & & & & & & & & \multicolumn{2}{|l|}{ Control } & \multicolumn{2}{|l|}{ Case } & \\
\hline & & & & & & & & Mean \pm SD & $n$ & Mean \pm SD & $n$ & \\
\hline 1998 & Liu & China & NA & Yes & Yes & SCC, ADC, LCC, SCLC & advanced & $6.0 \pm 0.5$ & 20 & $16.9 \pm 0.9$ & 28 & 7 \\
\hline 2001 & Koizumi & Japan & NA & NA & NA & SCC, ADC, LCC, SCLC & $\begin{array}{l}\text { early }+ \\
\text { advanced }\end{array}$ & $44 \pm 13$ & 11 & $77 \pm 47$ & 29 & 5 \\
\hline 2005 & Masri & USA & NA & NA & NA & $\begin{array}{l}\text { mixed (mainly SCC, } \\
A D C \text { ) }\end{array}$ & NA & $7.4 \pm 0.54$ & 35 & $18.4 \pm 3.16$ & 11 & 5 \\
\hline 2016 & $\mathrm{Xu}$ & China & Yes & Yes & Yes & SCC, ADC, SCLC & $\begin{array}{l}\text { early }+ \\
\text { advanced }\end{array}$ & $16.12 \pm 5.85$ & 51 & $25.60 \pm 16.93$ & 61 & 9 \\
\hline 2018 & Feng & China & Yes & Yes & Yes & SCC, ADC, SCLC & NA & $16.8 \pm 4.1$ & 50 & $33.8 \pm 15.6$ & 50 & 8 \\
\hline 2018 & Liu & China & Yes & Yes & NA & SCC, ADC, SCLC & NA & $16.83 \pm 4.17$ & 172 & $33.85 \pm 15.63$ & 164 & 8 \\
\hline
\end{tabular}

Abbreviations: NA Not available, NOS Newcastle-Ottawa Scale, SCC Squamous cell carcinoma, ADC Adenocarcinoma, LCC Large cell carcinoma, SCLC Small cell lung cancer 
Table 2 Characteristics of case-control studies about blood NO

\begin{tabular}{|c|c|c|c|c|c|c|c|c|c|c|c|c|}
\hline \multirow[t]{3}{*}{ Year } & \multirow[t]{3}{*}{ Author } & \multirow[t]{3}{*}{ Country } & \multirow{3}{*}{$\begin{array}{l}\text { Age } \\
\text { matched }\end{array}$} & \multirow{3}{*}{$\begin{array}{l}\text { Sex } \\
\text { matched }\end{array}$} & \multirow{3}{*}{$\begin{array}{l}\text { Smoking } \\
\text { matched }\end{array}$} & \multirow[t]{3}{*}{ Histology } & \multirow[t]{3}{*}{ Stage } & \multicolumn{4}{|c|}{ Blood NO ( $\mu \mathrm{mol} / \mathrm{L})$} & \multirow[t]{3}{*}{ NOS } \\
\hline & & & & & & & & \multicolumn{2}{|l|}{ Control } & \multicolumn{2}{|l|}{ Case } & \\
\hline & & & & & & & & Mean \pm SD & $\mathbf{n}$ & Mean \pm SD & $\mathbf{n}$ & \\
\hline 1996 & Ma & China & Yes & Yes & NA & $\begin{array}{l}\text { SCC, ADC, LCC, } \\
\text { SCLC }\end{array}$ & NA & $34.8 \pm 18.2$ & 34 & $48.5 \pm 15.8$ & 28 & 5 \\
\hline 1997 & Fan & China & Yes & Yes & NA & $\begin{array}{l}\text { mixed (mainly } \\
\text { SCC, ADC) }\end{array}$ & $\begin{array}{l}\text { early }+ \\
\text { advanced }\end{array}$ & $29.32 \pm 16.78$ & 25 & $58.75 \pm 19.63$ & 25 & 5 \\
\hline 1998 & $\mathrm{Li}$ & China & NA & Yes & NA & $\mathrm{SCC}, \mathrm{ADC}, \mathrm{SCLC}$ & $\begin{array}{l}\text { early }+ \\
\text { advanced }\end{array}$ & $42.10 \pm 17.22$ & 18 & $91.78 \pm 47.25$ & 25 & 6 \\
\hline 1998 & Yu & China & Yes & Yes & NA & $\mathrm{SCC}, \mathrm{ADC}$ & $\begin{array}{l}\text { early }+ \\
\text { advanced }\end{array}$ & $5.80 \pm 1.00$ & 30 & $7.11 \pm 1.35$ & 46 & 6 \\
\hline 1999 & Wu & China & Yes & Yes & NA & $\mathrm{SCC}, \mathrm{ADC}, \mathrm{SCLC}$ & $\begin{array}{l}\text { early }+ \\
\text { advanced }\end{array}$ & $29.6 \pm 11.4$ & 30 & $37.8 \pm 14.1$ & 30 & 7 \\
\hline 1999 & Yu & China & Yes & Yes & NA & $\mathrm{SCC}, \mathrm{ADC}$ & early & $5.82 \pm 1.31$ & 25 & $9.91 \pm 1.03$ & 18 & 6 \\
\hline 2001 & Bao & China & No & No & NA & $\mathrm{SCC}, \mathrm{ADC}, \mathrm{SCLC}$ & $\begin{array}{l}\text { early }+ \\
\text { advanced }\end{array}$ & $67.64 \pm 32.05$ & 40 & $53.22 \pm 26.08$ & 57 & 6 \\
\hline 2001 & Song & China & NA & Yes & NA & $\mathrm{SCC}, \mathrm{ADC}, \mathrm{SCLC}$ & $\begin{array}{l}\text { early }+ \\
\text { advanced }\end{array}$ & $3.45 \pm 0.94$ & 28 & $4.93 \pm 2.58$ & 30 & 6 \\
\hline 2001 & Zhu & China & No & No & NA & $\mathrm{SCC}, \mathrm{ADC}, \mathrm{SCLC}$ & $\begin{array}{l}\text { early }+ \\
\text { advanced }\end{array}$ & $84.69 \pm 12.10$ & 17 & $119.37 \pm 13.91$ & 40 & 5 \\
\hline 2001 & zheng & China & Yes & Yes & NA & $\begin{array}{l}\text { SCC, ADC, LCC, } \\
\text { SCLC }\end{array}$ & $\begin{array}{l}\text { early }+ \\
\text { advanced }\end{array}$ & $73.42 \pm 13.56$ & 30 & $49.42 \pm 18.78$ & 40 & 6 \\
\hline 2002 & Wu & China & NA & NA & NA & $\mathrm{SCC}, \mathrm{ADC}, \mathrm{SCLC}$ & $\begin{array}{l}\text { early }+ \\
\text { advanced }\end{array}$ & $85.54 \pm 39.63$ & 20 & $48.69 \pm 39.32$ & 40 & 5 \\
\hline 2002 & Wang & China & Yes & Yes & NA & $\mathrm{SCC}, \mathrm{ADC}$ & $\begin{array}{l}\text { early }+ \\
\text { advanced }\end{array}$ & $2.016 \pm 0.524$ & 36 & $5.902 \pm 1.157$ & 48 & 6 \\
\hline 2002 & Shi & China & NA & Yes & NA & NA & NA & $2.012 \pm 0.869$ & 29 & $6.952 \pm 2.571$ & 17 & 6 \\
\hline 2002 & Zhao & China & No & No & NA & $\mathrm{SCC}, \mathrm{ADC}$ & $\begin{array}{l}\text { early }+ \\
\text { advanced }\end{array}$ & $5.48 \pm 1.06$ & 60 & $7.38 \pm 2.15$ & 41 & 5 \\
\hline 2002 & Liu & China & No & Yes & NA & NSCLC, SCLC & $\begin{array}{l}\text { early }+ \\
\text { advanced }\end{array}$ & $49.3 \pm 17.9$ & 20 & $89.7 \pm 25.0$ & 70 & 6 \\
\hline 2002 & $\mathrm{Li}$ & China & No & Yes & NA & $\mathrm{SCC}, \mathrm{ADC}$ & NA & $5.24 \pm 1.18$ & 40 & $7.48 \pm 1.65$ & 30 & 6 \\
\hline 2003 & Liu & China & Yes & No & NA & $\mathrm{SCC}, \mathrm{ADC}$ & NA & $5.26 \pm 1.14$ & 50 & $8.65 \pm 2.06$ & 36 & 6 \\
\hline 2003 & Yu & China & Yes & Yes & Yes & $\mathrm{SCC}, \mathrm{ADC}$ & $\begin{array}{l}\text { early }+ \\
\text { advanced }\end{array}$ & $78.38 \pm 15.78$ & 30 & $105.71 \pm 25.68$ & 30 & 7 \\
\hline 2003 & Zhao & China & NA & Yes & NA & NA & early & $24.2 \pm 10.4$ & 35 & $60.4 \pm 27.2$ & 40 & 6 \\
\hline 2004 & Tang & China & NA & Yes & NA & SCC, ADC, LCC & NA & $76.81 \pm 16.61$ & 40 & $122.3 \pm 26.21$ & 51 & 7 \\
\hline 2005 & $\mathrm{Fu}$ & China & NA & Yes & NA & SCC, ADC, LCC & $\begin{array}{l}\text { early }+ \\
\text { advanced }\end{array}$ & $44.2 \pm 15.0$ & 20 & $237.1 \pm 21.0$ & 82 & 7 \\
\hline 2005 & Yin & China & Yes & Yes & NA & $\mathrm{SCC}, \mathrm{ADC}, \mathrm{SCLC}$ & $\begin{array}{l}\text { early }+ \\
\text { advanced }\end{array}$ & $4.77 \pm 1.81$ & 10 & $35.59 \pm 25.47$ & 41 & 6 \\
\hline 2005 & Chen & China & NA & NA & NA & NA & early & $68.5 \pm 14.7$ & 35 & $30.4 \pm 12.9$ & 31 & 5 \\
\hline 2005 & Kaynar & Turkey & Yes & Yes & NA & NSCLC, SCLC & $\begin{array}{l}\text { early }+ \\
\text { advanced }\end{array}$ & $43.4 \pm 9.3$ & 16 & $53.9 \pm 8.8$ & 32 & 6 \\
\hline 2006 & Colakogullari & Turkey & NA & NA & NA & NSCLC, SCLC & advanced & $63.7 \pm 32.2$ & 15 & $93 \pm 48$ & 31 & 6 \\
\hline 2006 & $\mathrm{Li}$ & China & Yes & Yes & NA & $\mathrm{SCC}, \mathrm{ADC}$ & $\begin{array}{l}\text { early }+ \\
\text { advanced }\end{array}$ & $54.6 \pm 16.4$ & 60 & $77.1 \pm 22.3$ & 62 & 6 \\
\hline 2008 & Yan & China & NA & No & NA & NA & NA & $23.67 \pm 6.18$ & 30 & $21.97 \pm 9.68$ & 40 & 5 \\
\hline 2008 & Esme & Turkey & Yes & No & Yes & SCC, ADC, LCC & $\begin{array}{l}\text { early }+ \\
\text { advanced }\end{array}$ & $145.93 \pm 27.03$ & 20 & $211.32 \pm 70.11$ & 49 & 7 \\
\hline 2009 & Long & China & NA & Yes & NA & SCC, ADC, LCC & NA & $77.81 \pm 17.61$ & 80 & $123.30 \pm 25.21$ & 100 & 6 \\
\hline 2010 & Srivastava & India & Yes & Yes & NA & mainly SCC, ADC, & advanced & $11.88 \pm 3.48$ & 144 & $25.76 \pm 4.54$ & 203 & 8 \\
\hline
\end{tabular}


Table 2 Characteristics of case-control studies about blood NO (Continued)

\begin{tabular}{lllllllllllllll}
\hline Year Author & Country & $\begin{array}{l}\text { Age } \\
\text { matched }\end{array}$ & $\begin{array}{l}\text { Sex } \\
\text { matched }\end{array}$ & $\begin{array}{l}\text { Smoking } \\
\text { matched }\end{array}$ & Histology & Stage & & \multicolumn{3}{l}{ Blood NO $(\boldsymbol{\mu m o l} / \mathbf{L})$} \\
\hline
\end{tabular}

Abbreviations: NA Not available, NOS Newcastle-Ottawa Scale, SCC Squamous cell carcinoma, ADC Adenocarcinoma, LCC Large cell carcinoma, NSCLC Non-small cell lung cancer, SCLC Small cell lung cancer

SCLC was higher than that in control patients. There was no significant difference of blood NO level among four kinds of lung cancer patients (Fig. 4c). The model ranked each pathological type by their relative effect (probabilities of being higher). According to these results, pathological type with the higher probability of being ranked first is LCC, with a probability of $67 \%$, followed by other three kinds of lung cancer, whose probability ranged from 10 to $12 \%$. Cumulative probability of being the pathological type with highest blood NO level was calculated and the cumulative ranking curve of each pathological type was obtained to calculate the SUCRA. According to SUCRA results, LCC (SUCRA = 83.5\%) presented as the cancer type with highest blood NO level and followed in order by ADC (58\%), SCC (56.5\%), SCLC (50\%) and control (1.5\%) (Table S3). The node-splitting approach allowed for the identification of two inconsistent nodes (SCC vs. SCLC, ADC vs. SCLC and LCC vs. SCLC). No inconsistent results of direct and indirect comparisons were observed in these three pairs of comparisons (Fig. 4e). In addition, BrooksGelman-Rubin plot illustrated that the NMA model presented good convergence.

There were 19 studies about the blood NO level of lung cancer patients in different stages (early or advanced) (Fig. 4b). The NMA model revealed that the blood NO level in advanced stage but not early stage was higher than that in control subjects (Fig. 4d). The model ranked each stage by their relative effect, and advanced stage was ranked first, with a probability of $91 \%$. According to SUCRA results, patients in advanced stage $($ SUCRA $=95.5 \%)$ had the highest blood NO level and

Table 3 Characteristics of studies about FeNO variation in lung cancer patients between pretreatment and posttreatment

\begin{tabular}{|c|c|c|c|c|c|c|c|c|c|c|c|c|c|}
\hline \multirow[t]{3}{*}{ Year } & \multirow[t]{3}{*}{ Author } & \multirow[t]{3}{*}{ Country } & \multirow{3}{*}{$\begin{array}{l}\text { Age } \\
\text { (Mean } \pm \\
\text { SD) }\end{array}$} & \multirow{3}{*}{$\begin{array}{l}\text { Sex } \\
\text { (M/ } \\
\text { F) }\end{array}$} & \multirow{3}{*}{$\begin{array}{l}\text { Smoking } \\
\text { status } \\
\text { (Mean } \pm \\
\text { SD) }\end{array}$} & \multirow[t]{3}{*}{ Histology } & \multirow[t]{3}{*}{ Stage } & \multirow[t]{3}{*}{ Treatment } & \multirow{3}{*}{$\begin{array}{l}\text { Follow } \\
\text { up } \\
\text { period }\end{array}$} & \multicolumn{4}{|c|}{ FeNO (ppb) } \\
\hline & & & & & & & & & & \multicolumn{2}{|l|}{$\begin{array}{l}\text { Before } \\
\text { therapy }\end{array}$} & \multicolumn{2}{|c|}{ After therapy } \\
\hline & & & & & & & & & & $\begin{array}{l}\text { Mean } \pm \\
\text { SD }\end{array}$ & $\mathbf{n}$ & $\begin{array}{l}\text { Mean } \pm \\
\text { SD }\end{array}$ & $\mathbf{n}$ \\
\hline 2001 & Koizumi & Japan & $\begin{array}{l}61^{a}(38- \\
84)\end{array}$ & $24 / 5$ & NA & $\begin{array}{l}\text { SCC, ADC, LCC, } \\
\text { SCLC }\end{array}$ & $\begin{array}{l}\text { early }+ \\
\text { advanced }\end{array}$ & radio-therapy & NA & $77.0 \pm 47.0$ & 29 & $49.0 \pm 33.0$ & 29 \\
\hline 2006 & Wewel & Germany & $64(12)^{b}$ & $\begin{array}{l}27 / \\
12\end{array}$ & $40(29)^{b}$ & NSCLC, SCLC & $\begin{array}{l}\text { early }+ \\
\text { advanced }\end{array}$ & $\begin{array}{l}\text { chemo- } \\
\text { therapy }\end{array}$ & $\begin{array}{l}21-29 \\
\text { days }\end{array}$ & $22.1 \pm 10.1$ & 34 & $24.5 \pm 10.8$ & 34 \\
\hline 2012 & Enache & France & $61 \pm 11$ & $\begin{array}{l}48 / \\
17\end{array}$ & NA & $\begin{array}{l}\text { SCC, ADC, LCC, } \\
\text { SCLC }\end{array}$ & $\begin{array}{l}\text { early }+ \\
\text { advanced }\end{array}$ & radio-therapy & $\begin{array}{l}7.5 \\
\text { months }\end{array}$ & $14.3 \pm 7.2$ & 65 & $18.2 \pm 12.5$ & 65 \\
\hline 2013 & Kallianos & Greece & $65 \pm 17$ & $\begin{array}{l}26 / \\
16\end{array}$ & $51 \pm 7$ & $\begin{array}{l}\text { SCC, ADC, LCC, } \\
\text { SCLC }\end{array}$ & NA & $\begin{array}{l}\text { chemo- } \\
\text { therapy }\end{array}$ & NA & $9.8 \pm 2.1$ & 42 & $7.7 \pm 1.6$ & 42 \\
\hline 2014 & Moré & USA & $\begin{array}{l}68^{\mathrm{a}}(54- \\
89)\end{array}$ & $14 / 6$ & NA & $\mathrm{NSCLC}$ & NA & radio-therapy & $\begin{array}{l}6 \\
\text { months }\end{array}$ & $26.3 \pm 19.6$ & 17 & $31.8 \pm 22.9$ & 17 \\
\hline 2019 & Szejniuk & Denmark & $\begin{array}{l}66^{\mathrm{a}}(40- \\
78)\end{array}$ & $\begin{array}{l}24 / \\
18\end{array}$ & NA & $\mathrm{NSCLC}$ & $\begin{array}{l}\text { early }+ \\
\text { advanced }\end{array}$ & radio-therapy & $\begin{array}{l}12 \\
\text { months }\end{array}$ & $13.5 \pm 7.6$ & 42 & $16.8 \pm 9.8$ & 42 \\
\hline 2019 & Suzuki & Japan & $\begin{array}{l}69^{a}(65- \\
74)\end{array}$ & $\begin{array}{l}74 / \\
21\end{array}$ & NA & $\mathrm{NSCLC}$ & advanced & $\begin{array}{l}\text { immune- } \\
\text { therapy }\end{array}$ & NA & $21.5 \pm 14.7$ & 85 & $25.2 \pm 16.8$ & 85 \\
\hline
\end{tabular}

Abbreviations: NA Not available, SCC Squamous cell carcinoma, ADC Adenocarcinoma, LCC Large cell carcinoma, NSCLC Non-small cell lung cancer, SCLC Small cell lung cancer

a Data presented as median (range)

${ }^{\mathrm{b}}$ Data presented as median (interquartile range) 
Table 4 Characteristics of studies about blood NO variation in lung cancer patients between pretreatment and posttreatment

\begin{tabular}{|c|c|c|c|c|c|c|c|c|c|c|c|c|c|}
\hline \multirow[t]{3}{*}{ Year } & \multirow[t]{3}{*}{ Author } & \multirow[t]{3}{*}{ Country } & \multirow{3}{*}{$\begin{array}{l}\text { Age } \\
\text { (Meean } \pm \\
\text { SD) }\end{array}$} & \multirow{3}{*}{$\begin{array}{l}\text { Sex } \\
\text { (M/F) }\end{array}$} & \multirow{3}{*}{$\begin{array}{l}\text { Smoking } \\
\text { status }\end{array}$} & \multirow[t]{3}{*}{ Histology } & \multirow[t]{3}{*}{ Stage } & \multirow[t]{3}{*}{ Treatment } & \multirow{3}{*}{$\begin{array}{l}\text { Follow } \\
\text { up } \\
\text { period }\end{array}$} & \multicolumn{4}{|c|}{ Blood NO ( $\mu \mathrm{mol} / \mathrm{L})$} \\
\hline & & & & & & & & & & \multicolumn{2}{|c|}{ Before therapy } & \multicolumn{2}{|l|}{ After therapy } \\
\hline & & & & & & & & & & $\overline{M e a n} \pm$ SD & $\mathbf{n}$ & $\overline{M e a n} \pm$ SD & $\mathrm{n}$ \\
\hline 1997 & Zhang & China & $58.4^{\mathrm{a}}$ & $13 / 7$ & NA & $\begin{array}{l}\text { SCC, ADC, LCC, } \\
\text { SCLC }\end{array}$ & advanced & chemo-therapy & 1 week & $51.2 \pm 16.9$ & 10 & $79.8 \pm 21.3$ & 10 \\
\hline \multirow[t]{2}{*}{1998} & \multirow[t]{2}{*}{ Yu } & \multirow[t]{2}{*}{ China } & \multirow[t]{2}{*}{$64.2^{\mathrm{a}}$} & \multirow[t]{2}{*}{ 28/18 } & \multirow[t]{2}{*}{ NA } & \multirow[t]{2}{*}{$S C C, A D C$} & \multirow{2}{*}{$\begin{array}{l}\text { early + } \\
\text { advanced }\end{array}$} & surgery & NA & $7.51 \pm 1.40$ & 26 & $6.68 \pm 1.19$ & 26 \\
\hline & & & & & & & & chemo-therapy & NA & $6.58 \pm 1.30$ & 15 & $5.43 \pm 1.43$ & 15 \\
\hline 1999 & Yu & China & $49 \pm 3.8$ & $14 / 4$ & NA & $S C C, A D C$ & $\begin{array}{l}\text { early }+ \\
\text { advanced }\end{array}$ & $\begin{array}{l}\text { chemo-therapy } \\
\text { /surgery }\end{array}$ & NA & $9.91 \pm 1.03$ & 18 & $8.48 \pm 1.06$ & 18 \\
\hline 2002 & Li & China & $\begin{array}{l}64.3^{\mathrm{b}} \\
(37-75)\end{array}$ & $53 / 31$ & NA & SCC, ADC & $\begin{array}{l}\text { early }+ \\
\text { advanced }\end{array}$ & chemo-therapy & NA & $64.6 \pm 29.5$ & 84 & $88.9 \pm 39.4$ & 84 \\
\hline 2002 & Wang & China & $65.4^{\mathrm{a}}$ & $35 / 13$ & NA & $\mathrm{SCC}, \mathrm{ADC}, \mathrm{LCC}$ & $\begin{array}{l}\text { early + } \\
\text { advanced }\end{array}$ & surgery & NA & $7.124 \pm 1.631$ & 26 & $6.094 \pm 0.917$ & 26 \\
\hline 2003 & Liu & China & $\begin{array}{l}61.4 \pm \\
11.3\end{array}$ & $30 / 6$ & NA & $S C C, A D C$ & NA & surgery & 10 days & $8.72 \pm 1.96$ & 20 & $6.83 \pm 1.75$ & 20 \\
\hline 2003 & Zhao & China & $51.8^{\mathrm{a}}$ & $28 / 12$ & NA & NA & early & surgery & 1 month & $60.4 \pm 27.2$ & 40 & $37.8 \pm 15.1$ & 40 \\
\hline 2005 & Yin & China & $\begin{array}{l}57.04 \pm \\
11.87\end{array}$ & $33 / 8$ & NA & $\mathrm{SCC}, \mathrm{ADC}, \mathrm{SCLC}$ & $\begin{array}{l}\text { early + } \\
\text { advanced }\end{array}$ & chemo-therapy & 2 weeks & $38.03 \pm 36.59$ & 40 & $29.33 \pm 20.97$ & 40 \\
\hline 2005 & Chen & China & NA & NA & NA & NA & early & surgery & $\begin{array}{l}3 \\
\text { months }\end{array}$ & $30.4 \pm 12.9$ & 31 & $62.2 \pm 15.1$ & 30 \\
\hline \multirow[t]{2}{*}{2006} & \multirow[t]{2}{*}{$\mathrm{Li}$} & \multirow[t]{2}{*}{ China } & \multirow[t]{2}{*}{$57.8^{\mathrm{a}}$} & \multirow[t]{2}{*}{$47 / 15$} & \multirow[t]{2}{*}{ NA } & \multirow[t]{2}{*}{ SCC, ADC } & \multirow{2}{*}{$\begin{array}{l}\text { early }+ \\
\text { advanced }\end{array}$} & surgery & 21 days & $86.7 \pm 24.1$ & 19 & $84.7 \pm 18.4$ & 19 \\
\hline & & & & & & & & chemo-therapy & 21 days & $89.4 \pm 22.1$ & 27 & $55.7 \pm 16.7$ & 27 \\
\hline 2006 & Colakogullari & Turkey & $\begin{array}{l}58.5 \pm \\
10.6\end{array}$ & $25 / 6$ & NA & NSCLC, SCLC & advanced & chemo-therapy & 2 days & $93.0 \pm 48.0$ & 31 & $106.0 \pm 49.0$ & 16 \\
\hline 2010 & Srivastava & India & $\begin{array}{l}55^{\mathrm{b}}(30- \\
88)\end{array}$ & $\begin{array}{l}155 / \\
48\end{array}$ & NA & $\begin{array}{l}\text { mainly SCC, } \\
\text { ADC, LCC }\end{array}$ & advanced & chemo-therapy & 88 weeks & $25.76 \pm 4.54$ & 203 & $32.81 \pm 4.27$ & 155 \\
\hline 2015 & $\mathrm{Xu}$ & China & $36-80^{c}$ & $24 / 12$ & NA & $\begin{array}{l}\text { SCC, ADC, LCC, } \\
\text { SCLC }\end{array}$ & NA & chemo-therapy & NA & $30.1 \pm 11.7$ & 36 & $62.0 \pm 14.7$ & 36 \\
\hline 2017 & Muto & Japan & $65.3 \pm 6.3$ & $6 / 9$ & NA & $A D C$ & advanced & chemo-therapy & $\begin{array}{l}>30 \\
\text { months }\end{array}$ & $62.7 \pm 40.0$ & 15 & $37.6 \pm 27.5$ & 15 \\
\hline \multirow[t]{2}{*}{2019} & \multirow[t]{2}{*}{ Feng } & \multirow[t]{2}{*}{ China } & $52.7 \pm 4.6$ & $28 / 15$ & NA & $S C C, A D C$ & advanced & chemo-therapy & $\begin{array}{l}3 \\
\text { months }\end{array}$ & $125.08 \pm 14.89$ & 43 & $89.77 \pm 11.35$ & 43 \\
\hline & & & $53.4 \pm 3.6$ & $26 / 18$ & NA & $S C C, A D C$ & advanced & $\begin{array}{l}\text { chemo-therapy + } \\
\text { TCM }\end{array}$ & $\begin{array}{l}3 \\
\text { months }\end{array}$ & $126.56 \pm 14.57$ & 44 & $78.12 \pm 8.12$ & 44 \\
\hline
\end{tabular}

Abbreviations: NA Not available, SCC Squamous cell carcinoma, ADC Adenocarcinoma, LCC Large cell carcinoma, NSCLC Non-small cell lung cancer, SCLC Small cell lung cancer

Data presented as mean value

Data presented as median (range)

'Data presented as range

followed in order by early stage $(52.5 \%)$ and control (2.5\%) (Table S4). The node-splitting approach allowed for the identification of two inconsistent nodes (early stage vs. advanced stage). No inconsistent results of direct and indirect comparisons were observed between these two groups (Fig. 4f). In addition, Brooks-GelmanRubin plot illustrated that the NMA model presented good convergence.

Blood NO level between pretreatment and posttreatment Fifteen observational studies including 18 cohorts reported blood NO level detected before and after therapy. As a result, no significant difference of blood NO level was observed between pretreatment and posttreatment (SMD -0.36, 95\% CI -1.08-0.36, $p>0.05$ ) (Fig. 3b). However, blood NO level decreased in NSCLC subgroup (SMD $-0.95,95 \%$ CI $-1.89-0.00, p=0.05$ ) after therapy. In other subgroups, there was no evident variance of blood NO level (Table S1).

\section{Publication bias}

Publication bias was tested using Begg's and Egger's tests. These tests did not show significant results in comparisons of FeNO. However, Begg's test and Egger's test showed significant results in the comparisons of blood NO level between cases and control subjects, and between pretreatment and posttreatment, respectively (Table S5). The distribution of data points revealed asymmetry (Fig. 5). These results indicated the possibility of publication bias.

\section{Discussion}

Lung cancer has becoming a great threat of global health due to its high incidence and mortality [62]. The fiveyear survival of this disease is relatively low, compared with other kinds of cancers [62]. A large proportion of patients were diagnosed when the lung cancer progressed to advanced stage. Much efforts have been made to diagnose lung cancer as early as possible. Tumor markers are molecules related to various types of tumors, which can be measured in blood as well as other biological samples $[5,63]$. The concentrations of these markers are very low in healthy population, but they can elevate dramatically in cancer patients. During the past years, several markers related to lung cancer were widely applied in clinic. However, the sensitivity and specificity were limited in the diagnosis of lung cancer. So, there is 


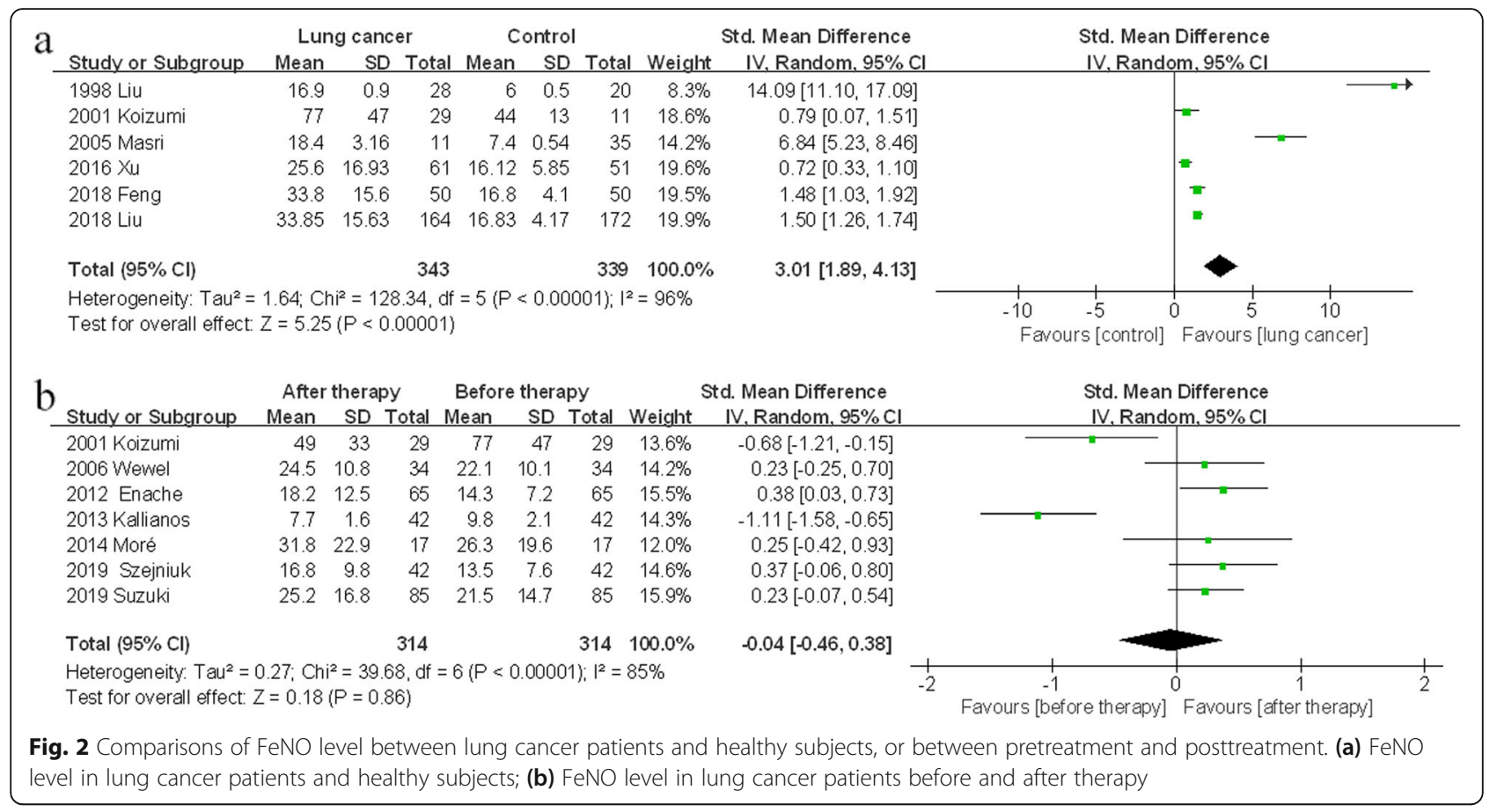

an urgent need for novel markers in diagnosing this disease, especially for those at early stage.

$\mathrm{NO}$ is a small molecule generated in many kinds of human cells. It was demonstrated that NO was involved in development of lung cancer in basic researches [3, 4, $64,65]$. Emerging studies has been carried out to evaluate whether NO can be regarded as a novel marker for lung cancer. However, controversial results were observed among these researches. In this meta-analysis, we summarized the previous data, and found FeNO value is much higher in lung cancer patients than that in control subjects, which meant an appropriate cut-off point of this index would be attributed to discriminate between lung cancer patients and healthy individuals. In some studies, the authors reported the FeNO values in different pathological types of lung cancer. Never the less, it was not further analyzed that whether the disparity of FeNO level existed among various types of lung cancer, due to the limited number of included literatures. Therefore, it is worthy to compare FeNO level in cancer patients with different pathological types in future studies. In contrast, a large proportion of included studies were about blood $\mathrm{NO}$ and lung cancer. As a result, an elevated blood NO level was found in lung cancer patients, which indicated this index was propitious for identifying lung cancer patients from general population. Subgroup analysis suggested ethnicity, sex, age and smoking status did not affect the result. However, the increase of blood NO level was only observed in the subgroup consisting of NSCLC patients, while in another subgroup containing SCLC patients, this phenomenon disappeared. It could be predicted that the rise of blood NO level existed in NSCLC but not SCLC patients, which was further confirmed by network meta-analysis. In addition, NMA results showed no difference in the comparisons of any two kinds of lung cancer types. LCC group seemed to be of highest blood NO value. However, the sample size of LCC group was rather small, subsequently, the results would be further validated in the future. NMA results also revealed that blood NO level in advanced stage but not early stage was higher than that in controls. Moreover, there was no difference of blood NO level between different stages. These results seemed to indicate that this index was less sensitive to capture lung cancer at early stage.

For lung cancer patients, proper treatments help to prolong survival time. Besides traditional treatments such as operation, chemotherapy and radiotherapy, a series of novel treatments have been developed, including molecular target therapy and immunotherapy [66]. In addition, traditional Chinese medicine is also an option of adjuvant therapy in China. As NO is considered to play an important role in lung cancer, the change of NO level between pretreatment and posttreatment has been drawn much attention. In this study, we compared both FeNO and blood NO data collected before and after therapy, and found almost no variance of NO level except for NSCLC and long-term subgroups. FeNO level escalated while blood NO level declined in NSCLC subgroup after treatment. Given that both FeNO and blood NO level increased in lung cancer patients, it seemed to be rather intriguing that the changing trends of FeNO 


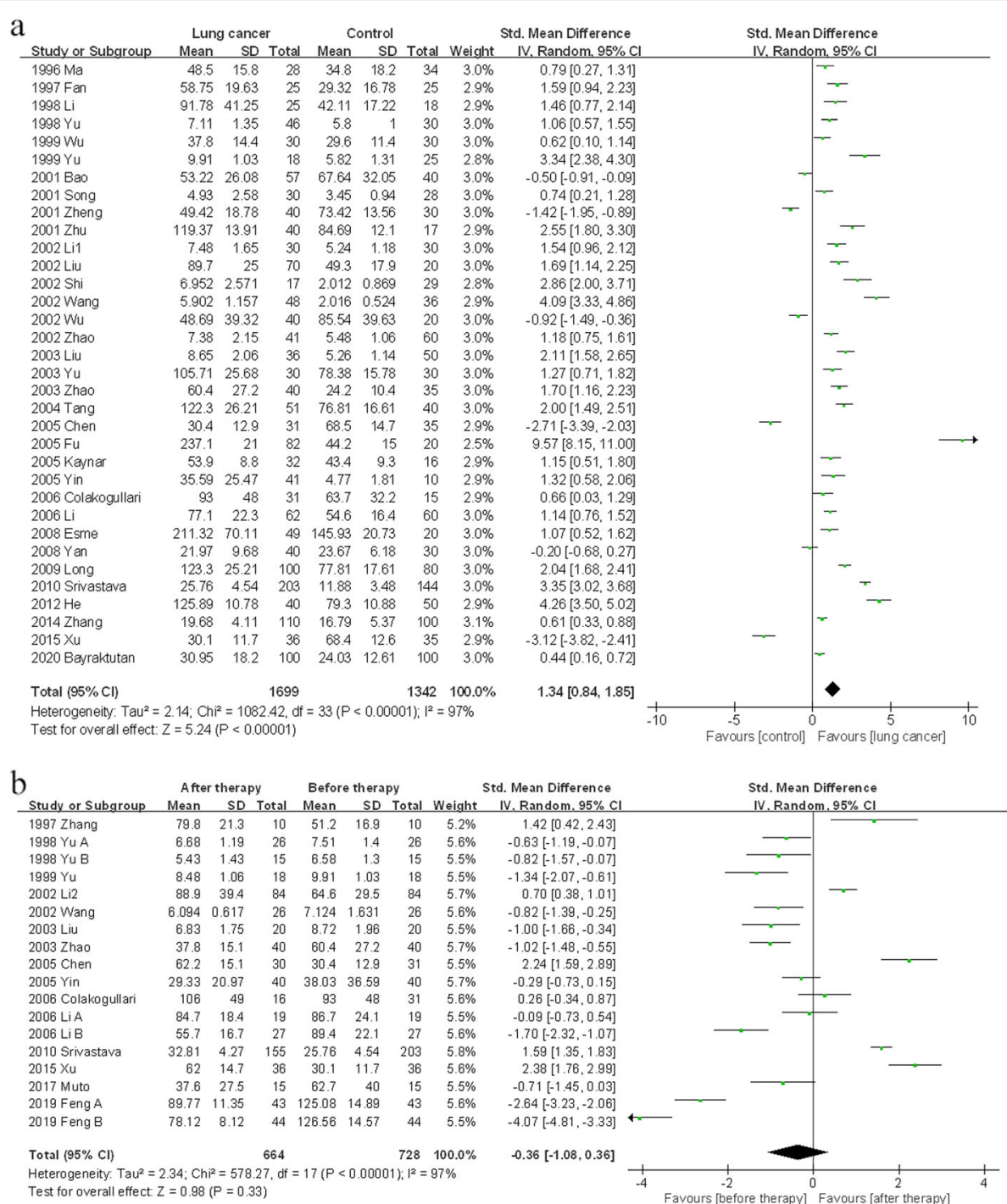

Fig. 3 Comparisons of blood NO level between lung cancer patients and healthy subjects, or between pretreatment and posttreatment. (a) Blood NO level in lung cancer patients and healthy subjects; (b) Blood NO level in lung cancer patients before and after therapy

level was just opposite to that of blood NO level after treatment. There were several reasons might lead to such results. First, it should be noted that patients in those two subgroups received different therapy regimens. The changing tendency of $\mathrm{NO}$ level was found to be inconsistent under different treatments. For example, Kallianos et al. found FeNO level decreased after chemotherapy [48], while Enache et al. reported FeNO level increased after radiotherapy [47]. So, it was necessary to detect both FeNO and blood NO levels in the same study to prevent the disturbance of therapy regimens. Another point that could not be overlooked was that there was great gap of follow up periods among different studies, from 2 days to more than 2 years $[7,10,14,46$, $47,52]$. Our results showed that FeNO level increased in long-term follow up subgroup but not in short-term follow up subgroup, while follow up period seemed not affect the pooled results of blood NO. In fact, NO value did not maintain the same level during the whole follow up period. According to the study by $\mathrm{Li}$ et al., the blood NO level decreased evidently 3 days after surgery, but increased slightly, and rose to the peak level, which is similar to that detected before therapy, 3 weeks after surgery [39]. In our study, we only chose the NO data collected at the last follow up time point, compared with that collected before therapy. Therefore, it was important to survey kinetic variation of NO level rather than observe $\mathrm{NO}$ value at a single time point. Last but not least, NO level would fluctuate under different disease status. As most studies included in our meta-analysis did not report the information of therapy effect or disease control, it could not be excluded that lung cancer 

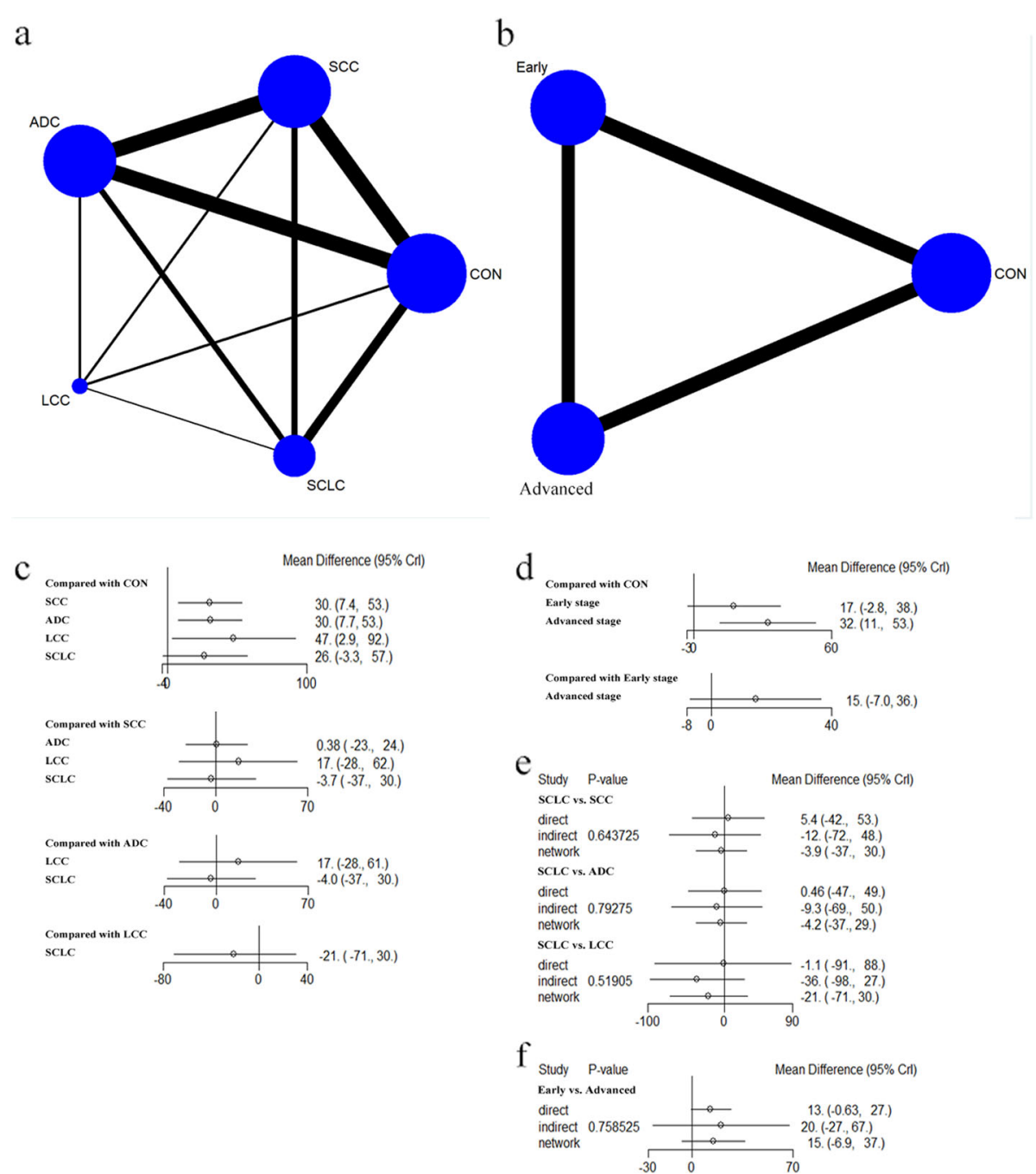

Fig. 4 Network meta-analysis of blood NO level in lung cancer patients with different pathological types and stages. (a) network plot of lung cancer patients with different pathological types; (b) network plot of lung cancer patients with different stages; (c) pairwise comparisons of blood NO level in controls and lung cancer patients with different pathological types; (d) pairwise comparisons of blood NO level in controls and lung cancer patients with different stages; (e) direct and indirect comparisons between two groups with different pathological types (SCC vs. SCLC, ADC vs. SCLC and LCC vs. SCLC); (f) direct and indirect comparisons between two groups with different stages (early stage vs. advanced stage)

progression offset the effect of previous therapy and finally affected the NO level, especially in patients undergone long-term follow up.

Our study had some deficiencies. First, the quantity of lung cancer patients in the included studies was few, especially for LCC patients. The small sample size might affect the stableness of the results. Second, a large proportion of included studies was of low quality, according to NOS. In some studies, the basic characteristics of control subjects (i.e. sex, age) were absent or not matched with lung cancer patients. The unmatched basic conditions might disturb the final results. Third, while most patients enrolled to study were Chinese, the remaining was consisting of other Asian and Caucasian. For Africans, lung cancer is also a common disease. It was regretful that almost no data on Africans was available. The incomprehensive data might affect the reliability of this study. Moreover, smoking is an important risk factor to the development of lung cancer, and it should be well controlled in these studies. However, a part of subjects in several studies have no information of smoking status. It might lead to the confusing results. Last but not least, NO is an indicator of airway inflammation in chronic airway diseases. It has been demonstrated that FeNO is elevated in COPD and asthma patients in previous studies [67-70]. COPD or asthma can be coexisted with lung cancer in some patients. For instance, Suzuki et al. and Wewel et al. reported part of lung cancer patients in their studies were combined with COPD [46, 51]. In contrast, lung cancer patients without 
a

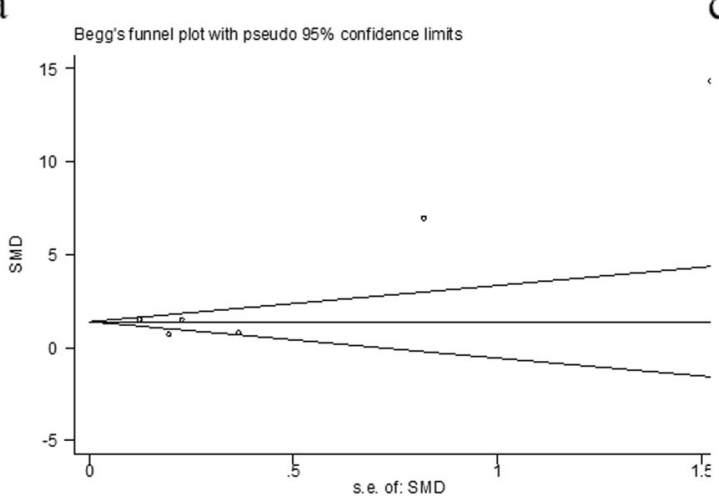

b

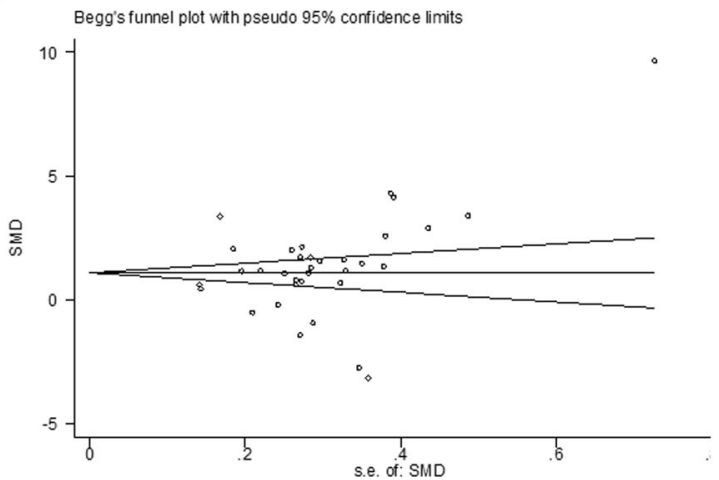

c

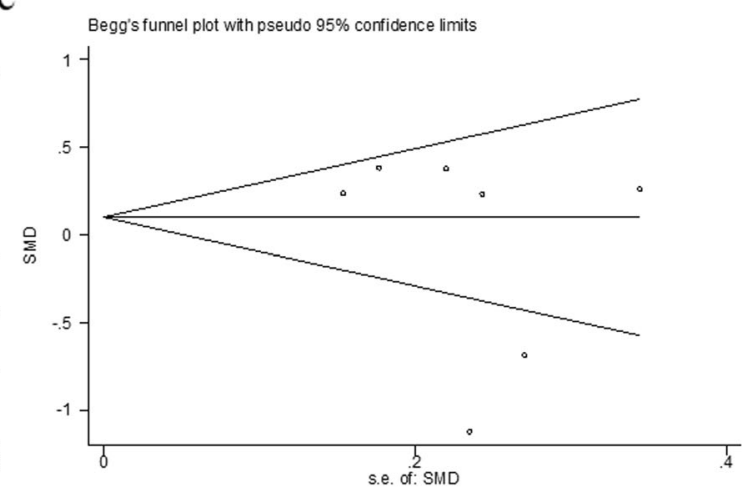

d

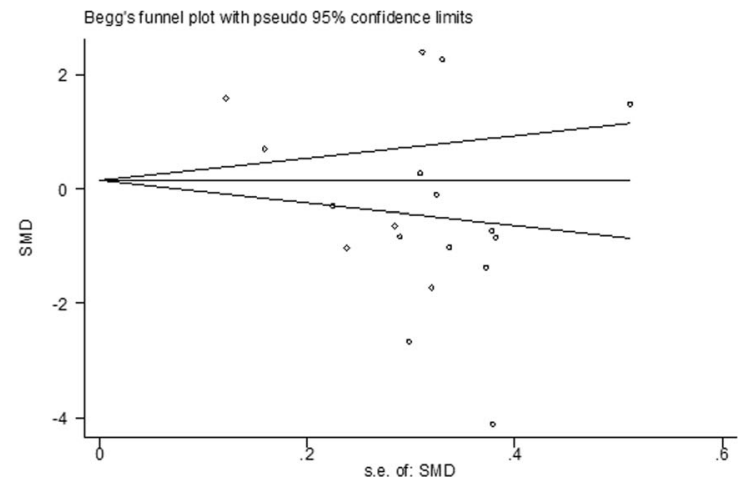

Fig. 5 Begg's funnel plot of comparisons between cases and controls or between pretreatment and posttreatment. (a) Begg's funnel plot of FeNO in case-control studies; (b) Begg's funnel plot of blood NO in case-control studies; (c) Begg's funnel plot of FeNO change in studies comparing FeNO level before and after therapy; (d) Begg's funnel plot of blood NO change in studies comparing blood NO level before and after therapy

comorbidities were recruited in several studies [12, 44, 45]. However, the majority studies did not provide the information that whether lung cancer patients had concomitant diseases. Concomitant disease may be a confounding factor which affected the final results. The deficiency of information about combined disease in lung cancer patients restricted us from further analysis.

\section{Conclusion}

In conclusion, we first demonstrated an evident augment of FeNO and blood NO level existed in whole lung cancer patients, but obvious change of these indexes was confirmed between pretreatment and posttreatment only in NSCLC patients. Our results suggested FeNO and blood NO level would contribute to diagnosis of lung cancer and evaluation of therapy effect, especially for NSCLC patients. Due to the various shortcomings in the present studies, future studies with a larger sample size, covering different pathological types and stages, and proper selection of control subjects should be carried out to further validate the relationship between $\mathrm{NO}$ and this disease.

\section{Abbreviations}

NO: Nitric oxide; FeNO: Fraction of exhaled nitric oxide; SMD: Standard mean difference; Cl: Confidence interval; NMA: Network meta-analysis;

SUCRA: Surface under the cumulative ranking curve; NSCLC: Non- small cell lung cancer; SCC: Squamous cell carcinoma; ADC: Adenocarcinoma; LCC: Large cell carcinoma; SCLC: Small cell lung cancer

\section{Supplementary Information}

The online version contains supplementary material available at https://doi. org/10.1186/s12885-021-08430-2.

\section{Additional file 1.}

\section{Acknowledgements}

Not applicable.

\section{Authors' contributions}

Zhou H, Li J and Ye S designed this study. Chen Z and Chen Y extracted the data of enrolled studies. Zhou $\mathrm{H}$ and $\mathrm{Li} I$ J were responsible for statistical analysis. Ye $S$ performed graph drafting. This manuscript was originally written and finally approved by all the authors.

\section{Funding}

This work was supported by grants from the National Natural Science Foundation of China (No. 81900029), Research Project of Public Welfare Technology Application of Zhejiang Province Science and Technology 
Department (No. LGC21H160004); Medical Science and Technology Project of Zhejiang Province (No. 2017KY196; 2020KY012).

\section{Availability of data and materials}

The datasets used in this study were available from the corresponding author upon reasonable request.

\section{Declarations}

\section{Ethics approval and consent to participate} Not applicable.

\section{Consent for publication}

Not applicable.

\section{Competing interests}

The authors declare no conflict of interest.

\section{Author details}

'Department of Respiratory Medicine, Department of Nutrition, Zhejiang Provincial People's Hospital, Affiliated People's Hospital, Hangzhou Medical College, 158 Shangtang Road, Hangzhou 310014, China. ${ }^{2}$ Department of Ophthalmology, Hangzhou Aier Eye Hospital, Zhejiang, Hangzhou, China. ${ }^{3}$ Department of Nutrition, Zhejiang Provincial People's Hospital, Affiliated People's Hospital, Hangzhou Medical College, Zhejiang, Hangzhou, China.

\section{Received: 17 February 2021 Accepted: 19 May 2021}

\section{Published online: 08 June 2021}

\section{References}

1. Siegel RL, Miller KD, Jemal A. Cancer statistics, 2020. CA Cancer J Clin. 2020; 70(1):7-30. https://doi.org/10.3322/caac.21590.

2. Bray F, Ferlay J, Soerjomataram I, Siegel RL, Torre LA, Jemal A. Global cancer statistics 2018: GLOBOCAN estimates of incidence and mortality worldwide for 36 cancers in 185 countries. CA Cancer J Clin. 2018:68(6):394-424. https://doi.org/10.3322/caac.21492.

3. Grimm EA, Sikora AG, Ekmekcioglu S. Molecular pathways: inflammationassociated nitric-oxide production as a cancer-supporting redox mechanism and a potential therapeutic target. Clin Cancer Res. 2013;19(20):5557-63. https://doi.org/10.1158/1078-0432.CCR-12-1554.

4. Zhan X, Huang Y, Qian S. Protein tyrosine nitration in lung Cancer: current research status and future perspectives. Curr Med Chem. 2018;25(29):343554. https://doi.org/10.2174/0929867325666180221140745

5. Zhou M, Liu Y, Duan Y. Breath biomarkers in diagnosis of pulmonary diseases. Clin Chim Acta. 2012;413(21-22):1770-80. https://doi.org/10.1016/j. cca.2012.07.006

6. Bayraktutan Z, Kiziltunc A, Bakan E, Alp HH. Determination of endothelial nitric oxide synthase gene polymorphism and plasma asymmetric dimethyl arginine concentrations in patients with lung Cancer. Eurasian J Med. 2020; 52(2):185-90. https://doi.org/10.5152/eurasianjmed.2020.19220.

7. Colakogullari M, Ulukaya E, Yilmaztepe A, Ocakoglu G, Yilmaz M, Karadag M, et al. Higher serum nitrate levels are associated with poor survival in lung cancer patients. Clin Biochem. 2006;39(9):898-903. https://doi.org/10.1016/j. clinbiochem.2006.06.008.

8. Esme H, Cemek M, Sezer M, Saglam H, Demir A, Melek H, et al. High levels of oxidative stress in patients with advanced lung cancer. Respirology. 2008; 13(1):112-6. https://doi.org/10.1111/j.1440-1843.2007.01212.x.

9. Kaynar H, Meral M, Turhan H, Keles M, Celik G, Akcay F. Glutathione peroxidase, glutathione-S-transferase, catalase, xanthine oxidase, cu-Zn superoxide dismutase activities, total glutathione, nitric oxide, and malondialdehyde levels in erythrocytes of patients with small cell and nonsmall cell lung cancer. Cancer Lett. 2005;227(2):133-9. https://doi.org/10.101 6/j.canlet.2004.12.005.

10. Koizumi M, Yamazaki H, Toyokawa K, Yoshioka Y, Suzuki G, Ito M, et al. Influence of thoracic radiotherapy on exhaled nitric oxide levels in patients with lung cancer. Jpn J Clin Oncol. 2001;31(4):142-6. https://doi.org/10.1 093/jjco/hye028.

11. Liu CY, Wang CH, Chen TC, Lin HC, Yu CT, Kuo HP. Increased level of exhaled nitric oxide and up-regulation of inducible nitric oxide synthase in patients with primary lung cancer. Br J Cancer. 1998;78(4):534-41. https:// doi.org/10.1038/bjc.1998.528.
12. Liu PF, Zhao DH, Qi Y, Wang JG, Zhao M, Xiao K, et al. The clinical value of exhaled nitric oxide in patients with lung cancer. Clin Respir J. 2018;12(1): 23-30. https://doi.org/10.1111/crj.12471.

13. Masri FA, Comhair SA, Koeck T, Xu W, Janocha A, Ghosh S, et al. Abnormalities in nitric oxide and its derivatives in lung cancer. Am J Respir Crit Care Med. 2005;172(5):597-605. https://doi.org/10.1164/rccm.200411-1 5230C.

14. Srivastava AN, Gupta A, Srivastava S, Natu SM, Mittal B, Negi MPS, et al. Cisplatin combination chemotherapy induces oxidative stress in advance non small cell lung Cancer patients. Asian Pac J Cancer P. 2010;11(2):465-71.

15. Zhang HY, Wu XW, Xiao Y, Chen M, Li ZD, Wei X, et al. Genetic polymorphisms of glutathione S-transferase M1 and T1, and evaluation of oxidative stress in patients with non-small cell lung cancer. Eur J Med Res. 2014;19(1):67. https://doi.org/10.1186/s40001-014-0067-3.

16. Ma X, Yang D, Zhang B, Ke B, Xu J. Detection and clinical significance of serum interleukin 8 and nitric oxide in lung cancer patients. Chin J Clin Lab Sci. 1996;14(4):190.

17. Fan $X M$, Zhan $X Q$, Xiong $Y$. Primary study on the association between serum nitric oxide level and lung cancer. Chin J Clin Oncol Rehabil. 1997; 4(4):25.

18. Li S, Zhang H, Zhang Z, Deng F, Zhang C. Nitric oxide and lung cancer immunity. Acta Univ Scientiae Med Chongqing. 1998;23(4):338-41.

19. Yu W, Zhang S, Du C, Liu S, Jiang H, Li D. Endogeneous nitric oxide level in serum of lung cancer and tuberculosis patients. Chin J Pract Intern Med. 1998;18(11):678-9.

20. Wu J, Dai S, Li Y, Yang Y. Primary study on the relationship between nitric oxide and elder patients with lung cancer. J Clin Pumlonary Med. 1999;4:15-6.

21. Yu L, Chen $X$, Gao F, Lv T, Zhang H, Zhang $Y$, et al. The change of serum nitric oxide cencentration in lung cancer patients. Tianjin Med J. 1999; 27(10):596-7.

22. Bao XW, Liu K, Cheng QS, Li XF. The changes and significance of nitric oxide and nitric oxide synthase in serum and tumor tissue of lung cancer patients. Acta Academiae Med CPAPF. 2001;10(1):10-3.

23. Song YM, Sun GQ, Yang WQ. Detection of nitric oxide concentration in serum and pleural fluid from lung cancer and tuberculous pleurisy patients. Chin J Lab Diagn. 2001;5(2):79-80.

24. Zheng S, Li H, Zhao J, Ge J, Shen Z. Significance of endothelin-1 and nitric oxide changes in serum and tumor tissue from lung cancer patients. Clin Med China. 2001;17(9):696-7.

25. Zhu SY, Wei CL, Lv XX. Expression of inducible nitric oxide synthase in lung cancer and its significance. Acta Acad Med Xuzhou. 2001;21(4):285-8.

26. $\mathrm{Li} \mathrm{ZH}$, Guan $\mathrm{LL}, \mathrm{Wu} \mathrm{HL}$. Detection and clinical significance of serum and tumor tissue nitric oxide in elder patients with lung cancer. Mod J Integr Tradit Chin West Med. 2002;11(22):2205-6.

27. Liu XD, Ge YJ, Yu J, Wei T. Clinical study of plasma calcitonin gene related peptide, nitric oxide and endothelin in lung cancer patients. Cap Med. 2002; 9:55-6.

28. Shi GL, He HM, Liu JB, Wang XY. Determination of serum nitric oxide and carcinoembryonic antigen in patients with lung Cancer and its clinical significance. Pract Clin Med. 2002;3(4):9-10.

29. Wang M, Wang J, Wang B, Gao M, Sun H, Wei W. Clinical significance of nitric oxide concentration of plasma in patients with pulmonary cancer. Pract J Card Cereb Pneumal Vasc Dis. 2002;10(3):140-1.

30. Wu YJ, Wu YM, Wang J, Qu LB, Xiang BR. Clinical significance of serum sIL$6 \mathrm{R}$ and NO detection in lung cancer patients. Tianjin Med J. 2002;30(2):73-5.

31. Zhao C, Liang B, Wu H. Detection and clinical significance of $\mathrm{NO}$ and NOS in lung cancer patients. Hunan Med J. 2002;19(2):97-8.

32. Liu YQ, Liang B, Guan LL, Wu HL. Study on immune functions of erythrocytes and serum NO/NOS in patients with lung cancer. J Clin Res. 2003;20(2):94-6.

33. Yu WH, Pan T, Qin Y, Lu XB, Liu SS, Luo Q, et al. A study of relationship between GST and NO of serum and lung Cancer in female non-smoker. China Cancer. 2003;12(4):212-4

34. Zhao $Y$, Jin TY. The significance of detecting the serum CEA and NO in patients with lung carcinoma. J Huaihai Med. 2003;21(6):442-3.

35. Tang RG, Tang XQ, Fang WZ. Clinical application of serum CEA and NO detection in lung cancer patients. J Youjiang Med Coll Nationnalities. 2004; 26(6):835.

36. Chen $\mathrm{H}$. Clinical significance of determination of changes of serum NO/ NOS, CEA, sIL-2R levels after operation in patients with lung cancer. $J$ Radioimmunol. 2005;18(5):346-7. 
37. Fu X, Huang Q, Sun W, Zhang N. Implication of serum leveis of interleukin18 and nitric oxide in tumor growth and metastasis of non-small cell lung cancer. Chin J Exp Surg. 2005;22(5):577-9.

38. Yin Y, Zhao W, Li F. Clinical significance of serum nitric oxide detection in lung cancer patients. Clin Focus. 2005;20(17):989-90.

39. Li QQ, Gan KD, Chen ZM. Clinical significance of serum NO and VEGF leve in lung cancer patients. Guangxi Med J. 2006;28(12):1900-2.

40. Yan Q, Miao G, Wang D, Cheng X, Pang D. Study on serum lipid peroxidation reaction in lung cancer patients. J Baotou Med Coll. 2008;24(4): $346-7$.

41. Long XK. The clinical applications of detecting the nitric oxide in Sernm of the patients with lung cancer. Lab Med Clin. 2009;6(19):1616 1618

42. He SY, Han HM, Wu HR, Li C. Clinical significance of serum vascular endothelial growth factor and nitric oxide level in non-small cell lung cancer patients. J Clin Intern Med. 2012;29(8):562-3.

43. Xu JC. Clillical Significance of Serum levels of NSE, NO/NOS, IGF-II in Lung Cancer Patients Before and After Chemotherapy. Labeled Immunoassays Clin Med. 2015;22(2):122-4.

44. Xu EW, Zhu QH, He Z, Qiao GB. The application of fraction exhaled nitric oxide in lung cancer diagnosis. Guangdong Med J. 2016;37(1):83-5.

45. Feng $\mathrm{T}, \mathrm{Li} \mathrm{H}$. The change and clinical significance of fraction exhaled nitric oxide value in lung cancer patients. Contemp Med. 2018;24(3):128-30.

46. Wewel AR, Crusius JAM, Gatzemeier U, Heckmayr M, Becher G, Magnussen $\mathrm{H}$, et al. Time course of exhaled hydrogen peroxide and nitric oxide during chemotherapy. Eur Respir J. 2006;27(5):1033-9. https://doi.org/10.1183/ 09031936.06.00101705

47. Enache I, Noel G, Jeung MY, Meyer N, Oswald-Mammosser M, Urban-

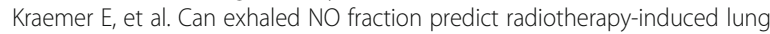
toxicity in lung cancer patients? Radiat Oncol. 2012;7(1):117. https://doi. org/10.1186/1748-717X-7-117.

48. Kallianos A, Tsimpoukis S, Zarogoulidis P, Darwiche K, Charpidou A, Tsioulis I, et al. Measurement of exhaled alveolar nitrogen oxide in patients with lung cancer: a friend from the past still precious today. Onco Targets Ther. 2013; 6:609-13. https://doi.org/10.2147/OTT.S44087.

49. More JM, Eclov NCW, Chung MP, Wynne JF, Shorter JH, Nelson DD Jr, et al. Feasibility and potential utility of multicomponent exhaled breath analysis for predicting development of radiation pneumonitis after stereotactic ablative radiotherapy. J Thorac Oncol. 2014;9(7):957-64. https://doi.org/10.1 097/JTO.0000000000000182.

50. Muto S, Takagi H, Owada $Y$, Inoue $T$, Watanabe $Y$, Yamaura $T$, et al. Serum nitric oxide as a predictive biomarker for bevacizumab in non-small cell lung Cancer patients. Anticancer Res. 2017;37(6):3169-74. https://doi.org/1 0.21873 /anticanres.11676.

51. Suzuki $Y$, Inui N, Karayama M, Imokawa S, Yamada T, Yokomura $K$, et al. Effect of PD-1 inhibitor on exhaled nitric oxide and pulmonary function in non-small cell lung cancer patients with and without COPD. Int J Chron Obstruct Pulmon Dis. 2019;14:1867-77. https://doi.org/10.2147/COPD.S21461 0 .

52. Szejniuk WM, Nielsen MS, Bronnum D, Takacs-Szabo Z, Weinreich UM, Pilegaard Thomsen $L$, et al. Fractional exhaled nitric oxide as a potential biomarker for radiation pneumonitis in patients with non-small cell lung cancer: a pilot study. Clin Transl Radiat Oncol. 2019;19:103-9. https://doi. org/10.1016/j.ctro.2019.09.004.

53. Zhang B, Liu S, Zhang S, Ma X. The effect of chemical immunotherapy on the nitric oxide in the body fluid from lung cancer patients. Med J Chin PLA. 1997;21(1):39.

54. Li P, Gu YQ, Yu FZ, Wu J, Zhang CH, Tan WR. The impact of chemotherapy on serum tumor markers and nitrogen monoxide in patients with non-small cell lung Cancer. J Chin Oncol. 2002;8(4):213-4.

55. Feng Y, Chen SN, Jiang Y, Zhou Y, Liao HF, Feng J, et al. Clinical and serological responses to Bufeihuayu decoction adjuvant therapy in patients with non-small cell lung Cancer. Chin Gen Pract. 2019;22(18):2223-9.

56. Wells GA, Shea B, O'Connell D, Peterson J, Welch V, Losos M, et al. The Newcastle-Ottawa Scale (NOS) for assessing the quality of nonrandomised studies in meta-analyses. http://www.ohri.ca/programs/clinical_ epidemiology/oxford.asp

57. Higgins JP, Thompson SG. Quantifying heterogeneity in a meta-analysis. Stat Med. 2002;21(11):1539-58. https://doi.org/10.1002/sim.1186.

58. Higgins JP, Thompson SG, Deeks JJ, Altman DG. Measuring inconsistency in meta-analyses. Bmj. 2003;327(7414):557-60. https://doi.org/10.1136/bmj.327. 7414.557.
59. Salanti G, Ades AE, loannidis JPA. Graphical methods and numerical summaries for presenting results from multiple-treatment meta-analysis: an overview and tutorial. J Clin Epidemiol. 2011;64(2):163-71. https://doi.org/1 0.1016/j.jclinepi.2010.03.016.

60. Begg CB, Mazumdar M. Operating characteristics of a rank correlation test for publication bias. Biometrics. 1994;50(4):1088-101. https://doi.org/10.23 07/2533446

61. Egger M, Davey Smith G, Schneider M, Minder C. Bias in meta-analysis detected by a simple, graphical test. Bmj. 1997;315(7109):629-34. https://doi. org/10.1136/bmj.315.7109.629.

62. Sun D, Li H, Cao M, He S, Lei L, Peng J, et al. Cancer burden in China: trends, risk factors and prevention. Cancer Biol Med. 2020;17(4):879-95. https://doi.org/10.20892/j.issn.2095-3941.2020.0387.

63. Chan HP, Lewis C, Thomas PS. Exhaled breath analysis: novel approach for early detection of lung cancer. Lung Cancer. 2009;63(2):164-8. https://doi. org/10.1016/j.lungcan.2008.05.020.

64. Iyer AK, Rojanasakul Y, Azad N. Nitrosothiol signaling and protein nitrosation in cell death. Nitric Oxide. 2014;42:9-18. https://doi.org/10.1016/j.niox.2014. 07.002 .

65. Luanpitpong S, Chanvorachote P. Nitric oxide and aggressive behavior of lung Cancer cells. Anticancer Res. 2015;35(9):4585-92.

66. Yuan M, Huang L-L, Chen J-H, Wu J, Xu Q. The emerging treatment landscape of targeted therapy in non-small-cell lung cancer. Signal Transduct Targeted Ther. 2019;4:61

67. Lu Z, Huang W, Wang L, Xu N, Ding Q, Cao C. Exhaled nitric oxide in patients with chronic obstructive pulmonary disease: a systematic review and meta-analysis. Int J Chron Obstruct Pulmon Dis. 2018;13:2695-705. https://doi.org/10.2147/COPD.S165780.

68. Lim CS, Rani FA, Tan LE. Response of exhaled nitric oxide to inhaled corticosteroids in patients with stable COPD: a systematic review and metaanalysis. Clin Respir J. 2018;12(1):218-26. https://doi.org/10.1111/crj.12518.

69. Wang Z, Pianosi PT, Keogh KA, Zaiem F, Alsawas M, Alahdab F, et al. The diagnostic accuracy of fractional exhaled nitric oxide testing in asthma: a systematic review and meta-analyses. Mayo Clin Proc. 2018;93(2):191-8. https://doi.org/10.1016/j.mayocp.2017.11.012.

70. Zhang L, Liu S, Li M, Xu X. Diagnostic value of fractional exhaled nitric oxide in cough-variant asthma: an updated meta-analysis. J Asthma. 2020;57(3): 335-42. https://doi.org/10.1080/02770903.2019.1568452.

\section{Publisher's Note}

Springer Nature remains neutral with regard to jurisdictional claims in published maps and institutional affiliations.
Ready to submit your research? Choose BMC and benefit from:

- fast, convenient online submission

- thorough peer review by experienced researchers in your field

- rapid publication on acceptance

- support for research data, including large and complex data types

- gold Open Access which fosters wider collaboration and increased citations

- maximum visibility for your research: over $100 \mathrm{M}$ website views per year

At BMC, research is always in progress.

Learn more biomedcentral.com/submissions 